\title{
Application of adjustment with contrainned codition in mixed GNSS, levelling control network and improving accuracy of the Geoid model
}

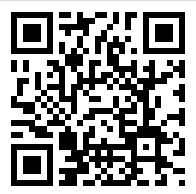

\section{Ha Ngoc Hoang *}

Faculty of Geomatics and Land Administration, Hanoi University of Mining and Geology, Vietnam

ARTICLE INFO ABSTRACT

Article history:

Received 05 ${ }^{\text {th }}$ Aug. 2020

Revised 23rd Sept. 2020

Accepted $31^{\text {st }}$ Oct. 2020

\section{Keywords:}

Adjustment computations,

Geoid model,

GNSS,

Height system,

Leveling networks.
One of the important contents to achieve the goal of modernization of the height system is connect the levelling observations between national levelling order I, II to the national GNSS Continuous Operating Reference Stations and the national gravity marks. Today, Vietnamese surveyors commonly use Geoid model 2010 that built based on more than 3000 gravity marks and over 800 GPS-levelling points. The processing combined GNSS-levelling and gravity obervations to improve the Geoid model for archiving accuracy from $4 \div 10$ centimeters, that can be possible to apply topographic heigh using GNSS accurately instead of traditional levelling is necessary for geomatics and mapping in Vietnam. There are some researchs in the literature aim to improving geoid model. In these research, the interpolation methods are mainly discussed such as collocation, linear function order 1, 2 or spline function. In this paper, the objective is to consider and propose a method of data processing using theothy of adjustment with contrainned codition and analyze the covariance matrix of the input data.

${ }^{*}$ Corresponding author

E-mail: hoangngocha@humg. edu.vn

DOI: 10. 46326/JMES. 2020.61(5). 07 


\section{Tạp chí Khoa học Kỹ thuật Mỏ - Địa chất}

\section{Ứng dụng phương pháp bình sai điều kiện kèm ẩn số trong xử lý hỗn hợp lưới GNSS , thủy chuẩn và nâng cao độ chính xác mô hình Geoid}

\section{Hoàng Ngọc Hà*}

Khoa Trắc địa - Bản đồ và Quản lý đất đai, Trường Đại học Mỏ - Địa chất, Việt Nam

\section{THÔNG TIN BÀI BÁO T TÓM TẮT}

Quá trình:

Nhận bài $05 / 8 / 2020$

Sưa xong 23/9/2020

Chấp nhận đăng 31/10/2020

\section{Tù̀ khóa:}

GNSS,

Hệ độ cao,

Lưới độ cao,

Mô hình Geoid,

Tính toán bình sai.
Một trong nhũng nội dung quan trọng để đạt mục tiêu hiện đại hóa hệ thống độ cao là tiến hành kết nối các tuyến độ cao lưới hạng İ, hạng II nhà nước với các điểm trạm GNSS CORS và các điểm trọng lực nhà nước. Hiện nay ở nước ta đang sử dung mô hình Geoid 2010 được xây dựng trên cơ sở mô hình Geoid toàn cầu EGM 2008 với bổ sung số liêu của trên 30.000 điểm trong lực chi tiết và trên 800 điểm GPS-thủy chuẩn. Việc xử lý kết hợp số liệu GNSSthủy chuẩn và mô hình Geoid trọng lực để nâng cấp mô hình Geoid địa phương đạt đô chính xác cao (cỡ $4 \div 10 \mathrm{~cm}$ ) có thể cho phép áp dụng công nghệ đo cao bẳng vệ tinh dần thay thế công nghệ đo thủy chuẩn truyền thống trong viêc xác định đô cao đat độ chính xác hang III và IV là bài toán cấp thiết của công tác trắc địa bản đồ ở nước ta. Về vấn đề xử lý số liệu nhằm nâng cấp mô hình Geoid đãa có nhiều tài liệu trong và ngoài nước đề cập. Trong các tài liệu này, chủ yếu thảo luận vấn đề áp dụng các mô hình cho hàm nội suy như phương pháp Collocation, hàm tuyến tính bậc 1, 2 hay hàm spline. Trong bài báo này, mục tiêu là xem xét và đề xuất bài toán xủ lý số liệu trắc địa tù̀ khía cạnh lý thuyết bình sai điều kiện kèm ẩn số và tính toán ma trận trọng số đảo của các số liệu đầu vào.

CC2020 Trường Đại học Mỏ - Địa chất. Tất cả các quyền được bảo đảm.

\section{Mở đầu}

Trên Hình 1 minh họa mối quan hệ giữa độ cao chuẩn và độ cao ellipsoid như phương trình (1)

$$
h=H-\zeta
$$

Trong đó: $h$ - độ cao chuẩn; $H$ - độ cao ellipsoid

\section{${ }^{*}$ Tác giả liên hệ}

E- mail: hoangngocha@humg.edu.vn DOI: 10. 46326/JMES. 2020. 61(5). 07 (độ cao trắc địa); $\zeta$ - dị thường độ cao.

Từ phương trình (1) ta có phương trình (2):

$$
H-\zeta-h=0
$$

Đại lượng $d \zeta=H-\zeta_{t l}-h=-F(x, y)$ hoặc $d \zeta=H-\zeta_{t l}-h=-F(B, L)$.

Mô hình bình sai 1

Có thể viết phương trình số hiệu chỉnh:

$$
V_{i}=a_{i} \Delta x_{i}+l_{i}
$$

Trong đó: $l_{i}=H_{i}-\zeta_{i}-H_{i}$ 


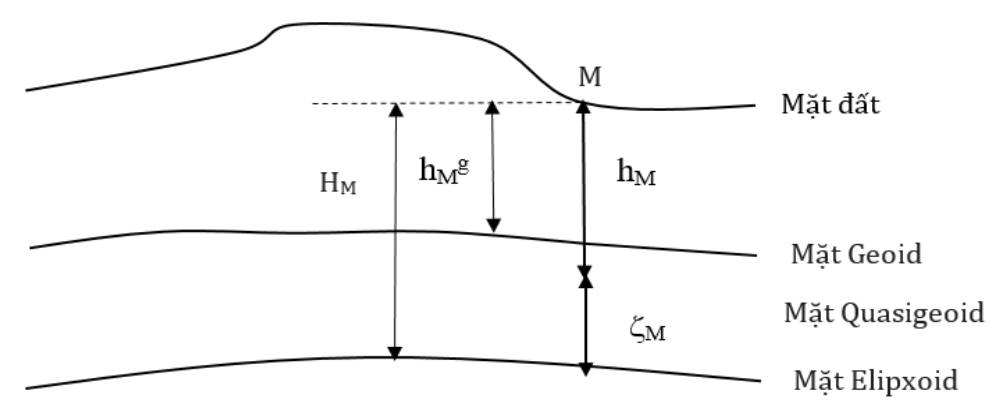

Hình 1. Mối quan hệ độ cao.

Thực tế có thể chọn mô hình 4 ẩn số (Hoàng Ngọc Hà, Trương Quang Hiếu, 2015; Markuze, Hoàng Ngọc Hà, 1991):

$$
\begin{gathered}
a_{i}=\left(\begin{array}{ccc}
1 & \cos B i \cos L i \cos B i \sin L i & \sin B i
\end{array}\right) \\
x^{T}=\left(\begin{array}{llll}
x_{0} & x_{1} x_{2} & x_{3}
\end{array}\right)
\end{gathered}
$$

Ở đây: $X_{0}, x_{1}, X_{2}, X_{3}$ - các hệ số của mô hình hàm nội suy.

Từ $n$ điểm GNSS có độ cao thủy chuẩn $h$, dị thường độ cao và độ cao trắc địa $H$, xác định hệ phương trình các số hiệu chỉnh:

$$
V=A \Delta_{x}+L
$$

Hệ phương trình chuẩn:

$$
R \Delta_{x}+b=0
$$

Giải hệ phương trình (6) xác định được $\Delta x$, và $x=x^{0}+\Delta x$. Như vậy, ta có các hệ số của mô hình (4).

Dị thường độ cao được tính toán từ các mô hình Geoid với các điểm không có độ cao thủy chuẩn:

$$
h=H_{G N S S}-\zeta+A x
$$

Nhược điểm của mô hình bình sai nay là không thể hiệu chỉnh các tham số $H$, $\zeta$ và $h$ sau bình sai.

\section{Mô hình bình sai 2}

Trong các tài liệu (Kotsakis và nnk, 2012; Lê Văn Hùng, Nguyễn Xuân Hòa, 2013) đã thảo luận vấn đề xử lý số liệu nhằm nâng cấp mô hình Geoid,trên cơ sở áp dụng các mô hình cho hàm nội suy như phương pháp Collocation, hàm tuyến tính bậc 1,2 hay hàm spline. Trong bài báo này xem xét bài toán xử lý số liệu trắc địa từ khía cạnh lý thuyết bình sai điều kiện kèm ẩn số mà các tác giả đã nêu ra ở (Hoàng Ngọc Hà, Markuze, 1990; Markuze, Hoàng Ngọc Hà, 1991; Hoàng Ngọc Hà, Trương Quang Hiếu, 2000; Hoàng Ngọc Hà, 2006; Leick và nnk. , 2015) và tính toán ma trận trọng số đảo của các số liệu đầu vào.

Với $n$ điểm GNSS có độ cao $H$ được xác định trong hệ tọa độ mặt đất và có độ cao thủy chuẩn, ta có các phương trình điều kiện với ẩn số phụ:

$$
\begin{gathered}
B V+A \Delta x+W=0 \\
B_{n x 3 n}=\left(\begin{array}{ccc}
E_{n x n}-E_{n x n}-E_{n x n}
\end{array}\right) \\
V^{T}=\left(\begin{array}{lll}
V_{H n x 1} & V \zeta_{n x 1} & E_{n x n}
\end{array}\right)
\end{gathered}
$$
số.

Trong đó: $E$ - Ma trận đơn vị; $A_{n \times k}$ - Ma trận hệ

$$
\begin{aligned}
& A^{T}=\left(\begin{array}{llll}
a_{1}^{T} & a_{2}^{T} & \ldots & a_{n}^{T}
\end{array}\right) \\
& Q_{V}=\left(\begin{array}{ccc}
Q_{H} & & \\
& Q_{\zeta} & \\
& & Q_{h}
\end{array}\right)
\end{aligned}
$$

Trong đó: $\Delta x$ - vector ẩn số; $Q_{H}, Q_{\zeta}, Q_{h}$ - ma trận trọng số đảo của các vector $H, \zeta, h$.

Đại lượng $W=H-\zeta-h$

$$
=(E-E-E)\left(\begin{array}{l}
H \\
\zeta \\
h
\end{array}\right)
$$

$$
Q_{V}=\left(\begin{array}{lll}
Q_{H} & & \\
& Q_{\zeta} & \\
& & Q_{h}
\end{array}\right)
$$

$$
\begin{aligned}
& Q_{y}=B Q B^{T} \\
& =(E-E-E)\left(\begin{array}{lll}
Q_{H} & & \\
& Q_{\zeta} & \\
& & Q_{h}
\end{array}\right)(E-E-E)^{T} \\
& =\left(Q_{H}+Q_{\zeta}+Q_{h}\right)
\end{aligned}
$$

Hệ phương trình (8) được giải với điều kiện:

$$
\begin{aligned}
\Phi=V^{T} Q_{V}^{-1} V= & V_{H}^{T} Q_{H}^{-1} V_{H}+V_{\zeta}^{T} Q_{\zeta}^{-1} V_{\zeta} \\
& +V_{h}^{T} Q_{h}^{-1} V_{h}=\min
\end{aligned}
$$


Từ lý thuyết bình sai điều kiện kèm ẩn số lập được hàm Lagrăng:

$$
\begin{gathered}
\Phi=V^{T} P V+2 K^{T}(B V+A \Delta x+W) \\
=\min
\end{gathered}
$$

Tính các đạo hàm theo vector:

$$
\begin{gathered}
\frac{\partial_{\varphi}}{\varphi V}=2 V^{T} P-2 K^{T} A=0 \\
\frac{\partial_{\varphi}}{\partial \Delta x}=-2 K^{T} A=0
\end{gathered}
$$

Từ công thức:

$$
\begin{gathered}
V=P^{-1} B^{T} K \\
A^{T} K=0
\end{gathered}
$$

Thay vào công thức (8) xác định được:

$$
\left\{\begin{array}{c}
N K+A \Delta x+W \\
A^{T} k=0 \\
N=B Q B^{T}=Q_{H}+Q_{\zeta}+Q_{\mathrm{h}}
\end{array}\right.
$$

Trong đó: $N=B Q B^{T} ; Q=P^{-1}$

Công thức (16) có thể viết lại dưới dạng:

$$
\left(\begin{array}{cc}
N & A \\
A^{T} & O
\end{array}\right)\left(\begin{array}{c}
K \\
\Delta x
\end{array}\right)+\left(\begin{array}{c}
W \\
O
\end{array}\right)=0
$$

Vector nghiệm của hệ phương trình (7) sẽ là:

$$
\left(\begin{array}{l}
K \\
\Delta x
\end{array}\right)=-B_{\beta}^{-1}\left(\begin{array}{l}
W \\
O
\end{array}\right)
$$

Ở đây, ma trận $N_{A}=\left(\begin{array}{cc}N & A \\ A^{T} & O\end{array}\right)$. Hệ phương trình (17) có thể được giải đơn giản hơn như sau. Từ phương trình đầu của hệ (16) có công thức:

$$
\begin{aligned}
K=-N^{-1} A \Delta x & -N^{-1} W \\
& =-N^{-1}(A \Delta x+W) \\
& =-N^{-1} W_{1}
\end{aligned}
$$

ở đây vector:

$$
W_{1}=A \Delta x+W
$$

Thay thế công thức (19) vào phương trình thứ 2 của hệ phương trình (16) sẽ nhận được:

Hay là:

$$
A^{T} N^{-1} A \Delta x+A^{T} N^{-1} W=0
$$

$$
\begin{aligned}
& \Delta x=-\left(A^{T} N^{-1} A\right)^{-1} A^{T} N^{-1} W \\
&=-\left[A ^ { T } \left(Q_{H}+Q_{\zeta}\right.\right. \\
&\left.+Q_{\mathrm{h}}\right)^{-1} A A^{T}\left(Q_{H}+Q_{\zeta}\right. \\
&\left.+Q_{\mathrm{h}}\right)^{-1} W
\end{aligned}
$$

Vector số hiệu chỉnh V được tính theo (14) và (19) như sau:

$$
\begin{aligned}
& V=\left(\begin{array}{l}
V_{H} \\
V_{\zeta} \\
V_{h}
\end{array}\right)=Q B^{T} K \\
& =-\left(\begin{array}{ccc}
Q_{H} & \\
& Q_{\zeta} & \\
& & Q_{h}
\end{array}\right)\left(\begin{array}{c}
E \\
-E \\
-E
\end{array}\right) N^{-1} W_{1} \\
& =-\left(\begin{array}{l}
Q_{H} \\
Q_{\zeta} \\
Q_{h}
\end{array}\right)\left(Q_{H}+Q_{\zeta}+Q_{h}\right)^{-1} W_{1}
\end{aligned}
$$

Để đánh giá độ chính xác sau bình sai, cần phải tính ma trận trọng số đảo của vector $V$ sau bình sai:

$$
Q_{v}=T Q w 1 T^{1}
$$

Ở đây ma trận:

$$
T=\left(\begin{array}{c}
Q_{H} \\
Q_{\zeta} \\
Q_{h}
\end{array}\right)\left(Q_{H}+Q_{\zeta}+Q_{h}\right)^{-1}
$$

Hoặc:

$$
T=\left(\begin{array}{c}
Q_{H} \\
Q_{\zeta} \\
Q_{h}
\end{array}\right) N^{-1}
$$

Ma trận trọng số đảo vector $x$ được xác định từ công thức (21):

$$
Q_{\Delta x}=\left(A^{T} N^{-1} A\right)^{-1}
$$

Ký hiệu ma trận $R=A^{T} N^{-1} A$ thay công thức (21) vào công thức (20) có:

$$
W_{1}=A \Delta x+W=\left(-A R^{-1} A^{T} N^{-1}+E\right) W
$$

Trong đó: $E$ - ma trận đơn vị.

Theo công thức tính trọng số đảo của hàm số có:

$$
\begin{gathered}
Q_{w 1}=\left(-A R^{-1}+E\right) N\left(-N^{-1} A R^{-1} A^{T}+E\right) \\
=\left(-A R^{-1} A^{T}+N\right)\left(-N^{-1} A R^{-1} A^{T}+E\right) \\
=A R^{-1} A^{T 1} A R^{-1} A^{T}-N^{-1} A R^{-1} A^{T}+A R^{-1} A^{T} \\
+N=N-A R^{-1} A^{T}
\end{gathered}
$$

Như vậy công thức:

$$
Q_{w 1}=N-A R^{-1} A^{T}
$$

Từ các kết quả tính toán theo công thức (21), vector $x$ dùng để tính độ cao chính đối với các 
điểm không có độ cao thủy chuẩn.

$V_{H i}, V_{\zeta}, V_{h i}$ sử dụng để hiệu chỉnh các giá trị $H_{i j}$ $\zeta_{i}, h_{i}$ tại các điểm GNSS và lưới độ cao.

Cần lưu ý rằng, trong bình sai hỗn hợp các đại lượng có tính chất khác nhau, thay vì sử dụng ma trận trọng số đảo nên dùng ma trận tương quan, được xác định theo định nghĩa:

$$
\begin{gathered}
C=\sigma^{2} Q \\
C_{V}=\left(\begin{array}{lll}
C_{H} & & \\
& C_{\zeta} & \\
& & C_{h}
\end{array}\right)
\end{gathered}
$$

Trong đó: $\sigma$ - Độ lệch chuẩn; $Q$ - ma trận trọng số đảo. Trong thực tế đại lượng $\sigma$ được thay thế bằng sai số trung phương trọng số đơn vị. Ma trận $Q$ được xác định từ kết quả bình sai riêng rẽ các mạng lưới trắc địa. Như vậy, để tối ưu hóa công việc tính toán các công thức từ (15) đến (25), các ma trận $Q_{H}, Q_{\zeta}, Q_{\mathrm{h}}$ được thay thế bằng ma trận $C_{H}, C_{\zeta}, C_{h}$.

\section{Tính toán thực nghiệm}

Để minh họa cho thuật toán đã trình bày ở trên, tác giả tiến hành tính toán với số liệu được đo thực nghiệm với sơ đồ như Hình 2. Các điểm 1, 2, 3, 4, 5 là những điểm nằm trong mạng lưới GNSS và thủy chuẩn. Các số liệu độ cao Geoid $\zeta$ và $C_{\zeta}$. được lấy từ mô hình Geoid hoặc được tính toán từ các số liệu đo trọng lực. Việc tính toán $\zeta$ được trình bày trong các tài liệu (Lê Văn Hùng, Nguyễn Xuân Hòa, 2013; Kotsakis và nnk., 2012). Việc xác định $C_{\zeta}$ để đưa vào tính toán có thể xác định độ lệch chuẩn thực nghiệm.

Bình sai riêng rẽ lưới độ cao và lưới GNSS được tính toán trên Ellipsoid WGS-84 đã được định vị để xác định các vector $h$, vector độ cao trắc địa $H$ và các ma trận $C_{h}$ và $C_{H}$. Các số liệu để đưa vào tính toán được đưa ở Bảng 1.

Ma trận A được thành lập như ma trận (*).

$$
\begin{aligned}
& A^{T} N^{-1} A=4 \cdot 10^{-3}
\end{aligned}
$$

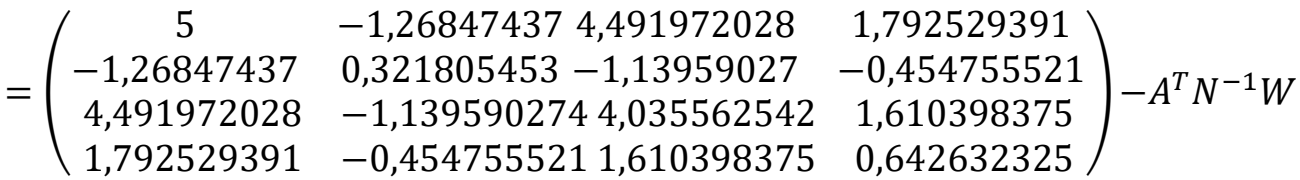

$$
\begin{aligned}
& =4 \cdot 10^{-3}\left(\begin{array}{c}
0,358 \\
-0,09082 \\
0,321625 \\
0,128346
\end{array}\right)
\end{aligned}
$$

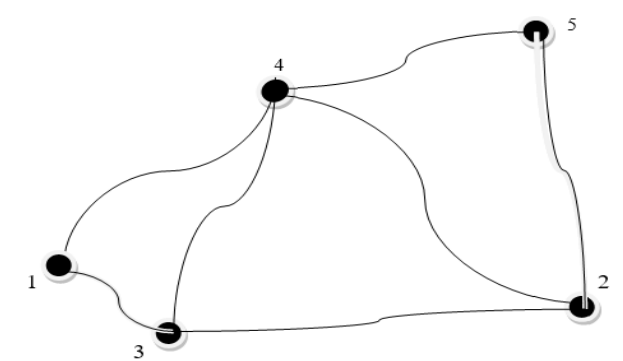

Hình 2. Sơ đồ các điểm chung GNSS và thủy chuẩn.

$$
A=\left(\begin{array}{llll}
1 & \cos B 1 \cos L 1 & \cos B i \sin L 1 & \sin B 1 \\
1 & \cos B 2 \cos L 2 & \cos B i \sin L 2 & \sin B 2 \\
1 & \cos B 3 \cos L 3 & \cos B 3 \sin L 3 & \sin B 3 \\
1 & \cos B 4 \cos L 4 & \cos B i \sin L 4 & \sin B 3 \\
1 & \cos B 4 \cos L 4 & \cos B i \sin L 4 & \sin B 4
\end{array}\right)
$$

Từ số liệu trong Bảng 1 , sẽ tính được các thành phần ma trận $A$ và vector $W$ trong Bảng 2 .

Ma trận:

$$
N=C_{H}+C_{\zeta}+C_{\mathrm{h}}
$$

Trong đó:

$$
C_{H}=\sigma H^{2} Q_{H} ; C_{\zeta}=\sigma \zeta^{2} Q_{\zeta} ; C_{h}=\sigma h^{2} Q_{h}
$$

Theo mô hình tính toán có:

$$
C_{H}=25 . \mathrm{E} ; C_{\zeta}=125 . E ; C_{h}=100 . E
$$

Nhận được ma trận (**).

Tính nghiệm $x$ theo công thức (21), vậy:

$$
\boldsymbol{X}=\left(\begin{array}{c}
-350404 \\
-86358,9 \\
313792,2 \\
129945,4
\end{array}\right)
$$

Vector $W_{1}$ trong công thức (20) được xác định như công thức (27):

$$
W_{1}=(A x+w)=\left(\begin{array}{c}
0,011995702 \\
-0,01206639 \\
0,001384377 \\
-0,001370179 \\
5,64605 E-05
\end{array}\right)
$$


Thực hiện công thức (22) tính được vector các số hiệu chỉnh $V$ :

$$
V^{T}=\left(V_{h 1} V_{h 2} \ldots V_{h n} V_{\zeta n} V_{h n} \ldots V_{n}\right)
$$

Từ công thức (23), ta có các tham số Bảng 3.

Từ kết quả tính toán các ma trận tương quan ở trên dễ dàng nhận thấy, sau khi bình sai tổng thể các thành phần đường chéo dùng để tính sai số trung phương các đại lượng $H_{i}, h_{i}, \zeta_{i}$ đều nhỏ hơn giá trị trước bính sai, ưu điểm của thuật toán là cho phép tiến hành xác định ma trận trọng số đảo sau bình sai tổng thể của các đại lượng đưa vào bình sai,cụ thể là $H, h$ và $\zeta$. Tác giả cũng đã áp dụng thuật toán để thử nghiệm với 19 điểm vùng Tây nguyên với số liệu lấy từ tài liệu của Lê Văn Hùng, Nguyễn Xuân Hòa (2013) và có kết quả là làm tăng độ chính xác các giá trị $\zeta$ và có thể dùng để nâng cấp mô hình Geoid địa phương.

Bảng 1. Dũ liệu đầu vào.

\begin{tabular}{|c|c|c|c|c|c|c|c|}
\hline \multirow{2}{*}{$\begin{array}{c}\text { Tên } \\
\text { điểm }\end{array}$} & \multicolumn{2}{|c|}{ Tọa độ phẳng } & \multicolumn{2}{|c|}{ Tọa độ trắc địa } & $\begin{array}{c}\text { Độ cao } \\
\text { trắc địa }\end{array}$ & $\begin{array}{c}\text { Độ cao } \\
\text { Geoid }\end{array}$ & $\begin{array}{c}\text { Độ cao thủy } \\
\text { chuẩn }\end{array}$ \\
\cline { 2 - 8 } & $X(\mathrm{~m})$ & $Y(\mathrm{~m})$ & $B\left(0^{\prime}{ }^{\prime \prime}\right)$ & $\left.L 0^{\prime}{ }^{\prime \prime}\right)$ & $H(\mathrm{~m})$ & $N(\mathrm{~m})$ & $H(\mathrm{~m})$ \\
\hline 1 & 2323837,548 & 579648,236 & 21026,08232 & 1054558,18666 & $-20,157$ & $-28,156$ & 7,977 \\
\hline 2 & 2324052,727 & 580277,877 & 21032,98045 & 1054620,02539 & $-20,909$ & $-28,148$ & 7,211 \\
\hline 3 & 2323878,279 & 579770,245 & 21027,38768 & 105462,41827 & $-20,871$ & $-28,154$ & 7,198 \\
\hline 4 & 2323932,544 & 579780,075 & 21029,15061 & 105462,76768 & $-20,983$ & $-28,155$ & 7,047 \\
\hline 5 & 2324169,145 & 580230,307 & 21036,77332 & 1054618,39762 & $-20,707$ & $-28,152$ & 7,347 \\
\hline
\end{tabular}

Bảng 2. Thành phần ma trận $A$ và vector $W$.

\begin{tabular}{|c|c|c|c|c|}
\hline \multicolumn{4}{|c|}{ Ma trân $A$} & Vector $W$ \\
\hline 1 & $-0,25365$ & 0,898414 & 0,35848 & $-0,022$ \\
\hline 1 & $-0,25374$ & 0,89838 & 0,35853 & $-0,028$ \\
\hline 1 & $-0,24843$ & 0,842034 & 0,47881 & $-0,085$ \\
\hline 1 & $-0,24869$ & 0,83766 & 0,48629 & $-0,125$ \\
\hline 1 & $-0,30477$ & 0,79908 & 0,51825 & $-0,098$ \\
\hline
\end{tabular}

Bảng 3. Ma trận tương quan sử dụng để đánh giá độ chính xác các tham số.

\begin{tabular}{|c|c|c|c|c|c|c|}
\hline TT & & & & lác tham số & & \\
\hline \multirow{5}{*}{1} & \multirow{5}{*}{$Q_{W_{1}}=$} & 121,7523596 & 20,30600433 & $-111,325858$ & $-33,59547132$ & $15,19614)$ \\
\hline & & & 5,095216293 & $-27,2525548$ & 3,734761623 & $-2,7352$ \\
\hline & & & & 137,4972685 & 7,489646814 & 0,649983 \\
\hline & & & & & 42,24054219 & $-14,5437$ \\
\hline & & 1 & & & & 8,438036 \\
\hline \multirow{5}{*}{2} & \multirow{5}{*}{$C_{H}=$} & 121,7523596 & 0,203060043 & $-1,11325858$ & $-0,335954713$ & 0,151961 \\
\hline & & & 0,050952163 & $-0,27252555$ & 0,037347616 & $-0,02735$ \\
\hline & & & & 1,374972685 & 0,074896468 & 0,0065 \\
\hline & & & & & 0,422405422 & $-0,14544$ \\
\hline & & & & & & $0,08438 /$ \\
\hline \multirow{5}{*}{3} & \multirow{5}{*}{$C_{\zeta}=$} & 60,87617981 & 10,15300216 & $-55,6629288$ & $-16,79773566$ & 7,598071 \\
\hline & & & 2,547608146 & $-13,6262774$ & 1,867380812 & $-1,3676$ \\
\hline & & & & 68,74863425 & 3,744823407 & 0,324991 \\
\hline & & & & & 21,12027109 & $-7,27186$ \\
\hline & & & & & & 4,219018 \\
\hline \multirow{5}{*}{4} & \multirow{5}{*}{$C_{h}=$} & (48,70094385 & 8,122401731 & $-44,5303431$ & $-13,43818853$ & 6,078457 \\
\hline & & & 2,038086517 & $-10,9010219$ & 1,493904649 & $-1,09408$ \\
\hline & & & & 54,9989074 & 2,995858726 & 0,259993 \\
\hline & & & & & 16,89621687 & $-5,81749$ \\
\hline & & & & & & 3,375215 \\
\hline
\end{tabular}


Để tính độ cao chuẩn của các điểm chỉ đo GNSS và không đo nối thủy chuẩn, áp dụng công thức:

$$
h_{j}=H_{j}-\zeta_{j}+a_{j} x
$$

Ở đây vector $x$ được tính trong công thức (26).

\section{Kết luận và kiến nghị}

Từ kết quả nghiên cứu lý thuyết và tính toán thực nghiệm nhận thấy, thuật toán bình sai hỗn hợp lưới thủy chuẩn, GNSS và số liệu từ mô hình Geoid hoặc đo trọng lực dựa trên cơ sở phương pháp bình sai điều kiện kèm ẩn số, với sự phát triển các công thức $(21 \div 25)$ đã được chứng minh cho phép bình sai chặt chẽ và sử dụng được kết quả bình sai riêng rẽ các lưới GNSS và thủy chuẩn. Thuật toán này có thể phục vụ công tác tính toán bình sai lưới trắc địa nhằm phục vụ việc hiện đại hóa hệ thống độ cao ở nước ta.

Thuật toán trên có thể ứng dụng trong trường hợp xử lý bài toán với số liệu là độ cao trắc địa, độ cao chính và độ cao Geoid.

\section{Tài liệu tham khảo}

Hoàng Ngọc Hà, (2006). Bình sai tính toán lưới Trắc địa và GPS. Nhà xuất bản Khoa học Kỹ thuật, Hà Nội.

Hoàng Ngọc Hà, Trương Quang Hiếu, (2000). Cơ sở toán học xử lý số liệu trắc địa. Nhà xuất bản Giao thông vận tải.

Kotsakis, C., Katsambalos, K., Ampatzidis, D., (2012). Estimation of the zero-height geopotential level in a local vertical datum from inver-sion of co-located GPS, levelling and geoid heights: a case study in the Hellenic islands. Journal of Geodesy 86(6), 423-439.

Lê Văn Hùng, Nguyễn Xuân Hòa, (2013). Kết hợp mô hình trọng trường toàn cầu EGM2008 và đo cao GPS thủy chuẩn nhằm nâng cao độ chính xác của kết quả đo cao GPS. Tạp chí Khoa học Công nghệ Xây dựng số 3+4.

Leick, A. , Rapoport, L., \& Tatarnikov, D., (2015). GPS satellite surveying. John Wiley \& Sons.

Markuze Y. U. I. , Hoàng Ngọc Hà, (1991). Bình sai các mạng lưới không gian mặt đất và vệ tinh, Nhà xuất bản Nhedra Matxcovva. Sách chuyên khảo (Tiếng Nga).

Markuze Y. I., (1990). Cơ sở bình sai tính toán bình sai. Nhà xuất bản Nhedra Moscow. 